Rolf W. Huegli

Anja-Carina Schulte

Markus Aschwanden

Christoph Thalhammer

Sebastian Kos

Augustinus L. Jacob

Deniz Bilecen

\section{Effects of percutaneous transluminal angioplasty on muscle BOLD-MRI in patients with peripheral arterial occlusive disease: preliminary results}

Received: 27 December 2007

Accepted: 7 August 2008

Published online: 16 September 2008

(C) European Society of Radiology 2008

R. W. Huegli · S. Kos · A. L. Jacob ·

D. Bilecen

Department of Radiology,

Division of Interventional Radiology,

University Hospital Basel,

Petersgraben 4,

4031 Basel, Switzerland

R. W. Huegli $(\bowtie)$

Department of Radiology,

Kantonsspital Bruderholz,

4101 Bruderholz, Switzerland

e-mail: rolf.huegli@ksbh.ch

Tel.: +41-61-4362320

Fax: +41-61-4363686

\section{A.-C. Schulte}

Biocenter, University of Basel,

4056 Basel, Switzerland

M. Aschwanden · C. Thalhammer

Department of Angiology,

University Hospital Basel,

Petersgraben 4,

4031 Basel, Switzerland
Abstract The purpose was to evaluate the effect of percutaneous transluminal angioplasty (PTA) of the superficial femoral artery (SFA) on the blood oxygenation level-dependent (BOLD) signal change in the calf musculature of patients with intermittent claudication. Ten patients (mean age, 63.4 \pm 11.6 years) with symptomatic peripheral arterial occlusive disease (PAOD) caused by SFA stenoses were investigated before and after PTA. Patients underwent BOLD-MRI 1 day before and 6 weeks after PTA. AT2*-weighted single-shot multi-echo echo-planar MR-imaging technique was applied. The BOLD measurements were acquired at mid-calf level during reactive hyperaemia at $1.5 \mathrm{~T}$. This transient hyperperfusion of the muscle tissue was provoked by suprasystolic cuff compression. Key parameters describing the BOLD signal curve included maximum $\mathrm{T} 2 *\left(\mathrm{~T} 2{ }^{*}{ }_{\max }\right)$, time-to-peak to reach $\mathrm{T}^{*}{ }_{\max }(\mathrm{TTP})$ and $\mathrm{T} 2 *$ end value (EV) after $600 \mathrm{~s}$ of hyperemia. Paired $t$ - tests were applied for statistic comparison. Between baseline and post-PTA, $\mathrm{T} 2{ }^{*}{ }_{\text {max }}$ increased from $11.1 \pm 3.6 \%$ to $12.3 \pm 3.8 \%(p=0.51)$, TTP decreased from $48.5 \pm 20.8 \mathrm{~s}$ to $35.3 \pm 11.6 \mathrm{~s}$ ( $p=$ $0.11)$ and $\mathrm{EV}$ decreased from $6.1 \pm$ $6.4 \%$ to $5.0 \pm 4.2 \%(p=0.69)$. In conclusion, BOLD-MRI reveals changes of the key parameters $\mathrm{T} 2{ }^{*}{ }_{\text {max }}$, TTP, and EV after successful PTA of the calf muscles during reactive hyperaemia.

Keywords Percutaneous transluminal angioplasty . Atherosclerosis $\cdot$ Muscle $\cdot$ Magnetic resonance imaging perfusion

\section{Introduction}

Arteriosclerosis is a slow progressive disease that causes accumulation of fatty substances, cholesterol, calcium, and fibrin forming plaque within the inner wall of the arteries $[1,2]$. Additionally, changes of the muscle tissue fiber type composition and architecture of microvasculature contributing to the impairment of blood supply were described [3-5]. The disease may be undiscovered as long as the blood supply is compensated by collateral pathways. A restriction in local perfusion can lead to symptoms like intermittent claudication up to gangrene of the extremities.

Depending on the clinical stage, a conservative treatment approach or revascularization may be indicated. The revascularization strategies involve percutaneous transluminal angioplasty (PTA) and open surgery. Presently, PTA is an established minimally invasive technique to treat vessel stenoses and/or occlusions by balloon dilation or stenting. Its beneficial effect on the macrocirculation is generally accepted and can be measured clinically by several methods 
including ankle-brachial index (ABI) or oscillography [6]. A drawback of these methods is their limited diagnostic value in case of incompressible calcified arteries [7].

Blood oxygenation level-dependent (BOLD) magnetic resonance imaging (MRI) of the human skeletal muscle was recently introduced as a new technique to describe the perfusion state of the lower limb musculature [8-11]. However, the mechanism of the muscle BOLD signal is not yet fully understood, but it is believed that the ratio of oxygenated to deoxygenated blood in the muscle tissue seems to play a major role for the BOLD response. It is also assumed that the mechanism is rather similar to that of the BOLD effect in the human brain $[10,11]$, where the brain regional hyperperfusion leads to an overproportional increase of oxyhemoglobin, which is accompanied by an increase of the BOLD signal [12-14].

Muscle BOLD is also dependent on muscle blood volume, perfusion vascular architecture and magnetic field angulation [15-17], but it seems to be more complex compared with the BOLD response in the brain.

First investigations of muscle BOLD MRI in peripheral arterial occlusive disease (PAOD) patients revealed significant changes of the muscle T2* BOLD time at calf level compared with a healthy age-matched control group [18]. These changes were referred to as an impairment of blood inflow and decrease in perfusion reserve capacity. The alterations of muscle tissue composition and microvascular architecture were also proposed as influencing factors. Therefore, a detectable BOLD signal change can be expected after endovascular therapy.

The purpose of our study was to compare the muscle BOLD response during reactive hyperemia in a calf muscle in patients suffering from intermittent claudication before and 6 weeks after PTA of the superficial femoral artery (SFA). A cuff-compression paradigm was applied.

\section{Materials and methods}

During an 8-month study period in 2006-2007, ten patients (63.4 \pm 11.6 years, eight male, two female) with intermittent claudication underwent antegrade PTA of the SFA. BOLD measurements in the gastrocnemius muscle of the calf were performed one day before and 6 weeks after PTA.

All PAOD-patients suffered from intermittent claudication with restriction of pain-free walking distance $<200 \mathrm{~m}$ and an ABI at rest of $<1$. Diagnostic pre-evaluation of the SFA was performed by duplex sonography (ATL HDI 5000; Philips, Best, The Netherlands). Only patients with up to two hemodynamic relevant SFA stenoses $>50 \%$ and a two- or three-vessel runoff of the infrapopliteal arteries were included in this study. Exclusion criteria were all common contraindications to MRI, like pacemakers, ferromagnetic implants and claustrophobia.

The study population's baseline characteristics are summarized in Table 1.
Table 1 Summary of clinical data

Patients $(n=10)$

\begin{tabular}{ll} 
Risk factors & \\
Smoking history & $8(80 \%)$ \\
Respiratory disease & $4(25 \%)$ \\
Arterial hypertension & $7(70 \%)$ \\
Hyperlipidemia & $8(80 \%)$ \\
Diabetes mellitus & $2(20 \%)$ \\
Medication & \\
Lipid-lowering agent & $7(70 \%)$ \\
Blood-pressure drug & $8(80 \%)$ \\
Heparin/vitamin K antagonist & $1(10 \%)$ \\
Aspirin/clopidogrel & $10(100 \%)$ \\
\hline
\end{tabular}

The study was approved by the local ethic committee and informed consent was obtained from every patient before entering the study.

\section{Cuff-compression paradigm for reactive hyperemia}

Reactive hyperemia was provoked using a cuff-compression paradigm $[18,19]$. The cuff $(15 \times 75 \mathrm{~cm})$ of a conventional leg-sphygmomanometer was fixed at mid-thigh level. Cuff compression with a pressure of $50 \mathrm{mmHg}$ above the individual brachial systolic blood pressure was applied during the ischemic phase for $240 \mathrm{~s}$. Cuff compression was performed manually and was adapted within $5 \mathrm{~s}$. BOLDMRI measurements started contemporaneously with cuff deflation and continued for $600 \mathrm{~s}$. Cuff deflation was initiated by fast opening of the air valve.

Digital subtraction angiography (DSA)-guided antegrade PTA

The patients were referred to the interventional unit of our radiological department for balloon angioplasty. The examinations were performed by two experienced interventional radiologists on a standard angiography unit (Multistar; Siemens Medical Systems, Erlangen, Germany). In the DSA unit, percutaneous vascular access was achieved by an antegrade puncture of the common femoral artery and a 4-F introducer sheath (Terumo Radifocus introducer II; Interleuvenlaan, Leuven, Belgium) was placed within the femoral artery.

To visualize the entire infrainguinal arteries including the SFA, deep femoral artery, popliteal artery, peroneal artery, anterior and posterior tibial artery diagnostic DSA with intraarterial injection of Ultravist 300 (Schering, Berlin, Germany) was acquired in four steps.

In all patients, a guidewire (V-18 control wire; Boston Scientific Medi-Tech, La Garenne Colombes Cedex, France) passage through the stenotic lesion was feasible. 
The lesions were treated by balloon angioplasty (Submarine Plus; Invatec Innovative Technologies, Roncadelle, Italy).

Balloon diameter varied between 4 and $5 \mathrm{~mm}$ and length ranged from 2 to $4 \mathrm{~cm}$. The results of angioplasty were documented by post-interventional DSA, which was also used to exclude thrombembolic complications below the knee. The puncture site was closed with a StarClose device (Abbott Vascular Devices, Abbott Laboratories, Redwood City, Calif.).

\section{MR-setup and BOLD measurements}

All MRI measurements were performed on a 1.5-T scanner (Sonata; Siemens Medical Solution, Erlangen, Germany). For signal detection a dedicated peripheral vascular array coil was used. Patients were investigated in the supine position.

A fat-saturated single-shot multi-echo echo-planar imaging (EPI) sequence with a temporal resolution of one acquisition per second was applied for muscle BOLD measurements. Four axial slices with a slice thickness of $5 \mathrm{~mm}$ and an imaging gap of $2.5 \mathrm{~mm}$ were positioned in the upper calf at maximal diameter. Acquisition parameters were as follows: $380 \times 238 \mathrm{~mm}$ field of view; $128 \times 80$ matrix size; $1,000 \mathrm{~ms}$ repetition time; four echo-images with effective echo times $\left(\mathrm{TE}_{\mathrm{eff}}\right)$ of $16,38,61,83 \mathrm{~ms}$. EPI images were referenced to anatomical $\mathrm{T}_{1}$-weighted spinecho images.

\section{Data analysis}

Oxygenation $\left(\mathrm{T}_{2} *\right)$ and initial signal intensity $\left(\mathrm{I}_{0}\right)$ effects were separated by a pixel-by-pixel least-square fit of monoexponential decay to the signal intensities of the echo-images using a postprocessing software implemented on our MR scanner. Parameter maps of T2* were generated from the multi-echo EPI data according to

$S\left(I_{0}, T_{2}^{*}\right)=I_{0} \cdot \exp \left(-T E_{\text {eff }} / T_{2}^{*}\right)$,

where $I_{0}$ refers to the initial signal intensity, which is modulated by perfusion, proton density, and $\mathrm{T}_{1}$ [20].

Based on the $\mathrm{T} 2 *$-maps, a region-of-interest (ROI) analysis was performed using the statistical parametric mapping software BrainVoyager (Brain Innovation, Maastricht, Netherlands).

A T2* time-course was extracted from a rectangular ROI with $\sim 100$ pixels within the gastrocnemius muscle. Pixels containing larger arteries and veins were excluded.

Data analysis was performed using self-developed Matlab (Mathworks, Natick, Mass., USA) routines. The data was averaged over the four acquired slices and normalized with respect to the average $\mathrm{T} 2 *$ value during the first three seconds of the measurement, i.e., after cuff deflation. Three key parameters were determined: maximum $\mathrm{T} 2 *\left(\mathrm{~T} 2 *_{\max }\right)$, time to peak (TTP), i.e., the elapsed time between cuff deflation and $\mathrm{T} 2{ }^{*}{ }_{\max }$, and the $\mathrm{T} 2 *$ end value $(\mathrm{EV})$ - the mean $\mathrm{T} 2 *$ of the last $2 \mathrm{~s}$ of the entire measurement lasting $600 \mathrm{~s}$ relative to the baseline. These muscle BOLD parameters were calculated for each of the patients before and 6 weeks after PTA. Paired $t$-tests were performed to compare pre- and post PTA key parameters T2* ${ }_{\max }$, TTP and EV and for the ABI values before and after interventions. A $p$ value $<0.05$ was considered as significant. No adjustments of $p$ values for multiple comparisons were done. Statistical analyses were carried out with SPSS (ver. 15).

\section{Results}

The i.a. DSA with PTA and the muscle BOLD procedure were well tolerated by all patients. DSA images acquired after PTA demonstrated a patent SFA or a stenosis grade $<25 \%$. In seven patients, a three-vessel run-off and in three patients a two-vessel run-off below the knee was observed. No thrombembolic complications occurred and the infrapopliteal run-off remained unchanged.

The ABI before PTA - averaged over all patients - was $0.74 \pm 0.10$ at rest and $0.51 \pm 0.14$ after exercise $(p=0.001)$. Six weeks after the intervention the mean $\mathrm{ABI}$ increased to $0.92 \pm 0.14$ at rest and $0.77 \pm 0.22$ after exercise $(p=0.002)$. The ABI improved on average by $>0.15$, reflecting the therapeutic success of the PTA treatment (Table 2). One patient was excluded due to incompressibility of calcified arteries and false high values in $\mathrm{ABI}$ measurements.

\section{Muscle BOLD response during reactive hyperemia}

Figure 1 illustrates the mean normalized $\mathrm{T} 2 *$ time-course in the gastrocnemius muscle of PAOD patients $(n=10)$ during $600 \mathrm{~s}$ of post-occlusive reactive hyperemia before (gray) and after PTA (black). Comparing the T2* timecourses, differences of the key parameters were detected. Firstly, an acceleration of BOLD peaking was observed in the post-PTA group, which is reflected by a decline of TTP from $48.5 \mathrm{~s} \pm 20.8 \mathrm{~s}$ to $35.3 \mathrm{~s} \pm 11.6 \mathrm{~s}(p=0.11)$. Secondly, the $\mathrm{T} 2{ }_{\max }$ amplitude increased from $11.1 \% \pm 3.6 \%$ to $12.3 \% \pm 3.8 \%(p=0.51)$ after treatment. And finally, the post-interventional muscle BOLD response showed a larger decrease after peaking, leading to a smaller EV of $5.0 \mathrm{~s} \pm 4.2 \mathrm{~s}$ compared with $6.1 \mathrm{~s} \pm 6.4 \mathrm{~s}(p=0.69)$. The BOLD data are presented in Table 2.

Figure 2a demonstrates the results of PTA and BOLD response of an 80-year-old patient. At the top, pre- and post-interventional DSAs of the right SFA are demonstrated. The short distance stenosis of the SFA was dilated 
Table 2 Summary of BOLD-MRI data

\begin{tabular}{lllll}
\hline & & Pre-PTA & Post-PTA & $p$ value \\
\hline$n=10$ & T2 ${ }_{\text {max }}(\%)$ & $11.1 \pm 3.6$ & $12.3 \pm 3.8$ & $>0.05$ \\
& TTP (s) & $48.5 \pm 20.8$ & $35.3 \pm 11.6$ & $>0.05$ \\
$n=9^{\mathrm{a}}$ & EV (\%) & $6.1 \pm 6.4$ & $5.0 \pm 4.2$ & $>0.05$ \\
& ABI at rest & $0.74 \pm 0.10$ & $0.92 \pm 0.14$ & $<0.05$ \\
\hline
\end{tabular}

${ }^{a}$ Due to the incompressibility of the arteries, the ABI-values of one patient were not available

using a 5/40-mm balloon catheter. A small not flow hampering dissection within the region of angioplasty was observed; however, the remaining stenosis was $<25 \%$. The corresponding $\mathrm{T}^{*}$ time-courses of the muscle BOLD signal pre- and post-PTA are shown at the bottom. Both time-courses show a steep increase in $\mathrm{T} 2 *$ after cuff deflation. This is followed by a slow decline after peaking at TTP until the T2* value almost remains constant. The T2* time-course after PTA displays a slightly accelerated TTP with an earlier peaking compared to the T2* timecourse before PTA. In addition, the post-interventional BOLD response shows an increase of $\mathrm{T} 2{ }^{*}{ }_{\text {max }}$. The ABI of this patient revealed a substantial increase from 0.60 to 0.88 after PTA at rest. Another PAOD patient is demonstrated in Fig. 2b after PTA of a stenotic tandem lesion in the SFA. The BOLD curve demonstrates clearly an acceleration of $\mathrm{T} 2 *$ peaking.

\section{Discussion}

The BOLD effect in the skeletal muscle is still an underinvestigated field of research. Furthermore, the muscle BOLD effect is presently not fully understood but it is believed that the muscle BOLD signal reflects-at least partially - the perfusion state of the muscle tissue [9$11,16,17]$.

The muscle BOLD response during reactive hyperemia has been described recently in PAOD patients suffering from intermittent claudication symptoms [18]. A delay in BOLD peaking (TTP) during reactive hyperemia was described. Additionally, a reduction of the $\mathrm{T} 2{ }^{*}{ }_{\max }$ was observed and the BOLD signal recovery (EV) was significantly delayed. It was assumed that the impairment of blood inflow due to atherosclerotic lesions was predominantly responsible for these effects [18].

So far, the influence of an underlying vascular disease and its impact on the muscle BOLD response after revascularization PTA-treatment has not yet been elucidated. In our study, we investigated symptomatic PAOD patients suffering from intermittent claudication with BOLD MRI before and 6 weeks after PTA treatment. In general, it is well accepted that stenosis treatment improves the macro- and microcirculation of the dependent tissue. Therefore, post-interventional changes of muscle BOLD response are expected. To go one step further, we hypothesized that the PTA treatment might reverse the PAOD-induced changes back to a normalization of TTP acceleration, increase in $\mathrm{T} 2{ }^{*}{ }_{\max }$ and BOLD recovery (EV) acceleration.

Blood inflow toward the calf tissue is certainly optimized by PTA. The blood flow through the original superficial femoral artery is faster than through the collaterals. A post-interventional decrease of TTP values can therefore be expected and was confirmed in this study. The acceleration was in the time range of $\sim 12 \mathrm{~s}(p=0.11)$. However, this finding was not significant, but demonstrates hemodynamic changes.

The elimination of stenotic lesions does also explain - at least partially - the increase of maximal $\Delta \mathrm{T} 2 *$ after intervention and might be explained by the optimization of blood oxygen supply after PTA treatment. This effect was recently demonstrated by transcutaneous oxygen pressure measurements $\left(\mathrm{TcPo}_{2}\right)$, where an improvement of tissue oxygenation is associated with an increase of oxyhemoglobin concentration in the capillary bed [21, 22]. It shifts the $\mathrm{Hb}_{\text {ox/deox }}$ ratio toward higher concentration. This increases the diamagnetic properties of the blood and raises the $\Delta \mathrm{T} 2 *$ signal. This is also supported by a muscle BOLD study in healthy volunteers, where good correla-

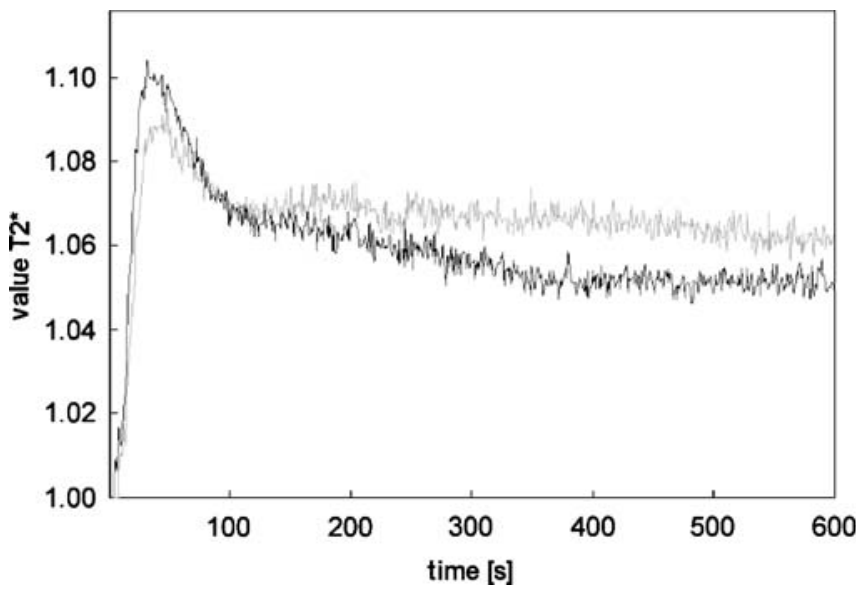

Fig. 1 Mean normalized muscle BOLD response before (gray) and after (black) successful PTA of the SFA obtained from ten patients suffering from claudication symptoms. In general, the postinterventional $\mathrm{T} 2 *$ time-course reveals a higher $\mathrm{T} 2 *$ max , a shorter TTP, and a lower EV than the pre-interventional time-course. The differences of the key parameters were not significant $(p>0.05)$ 

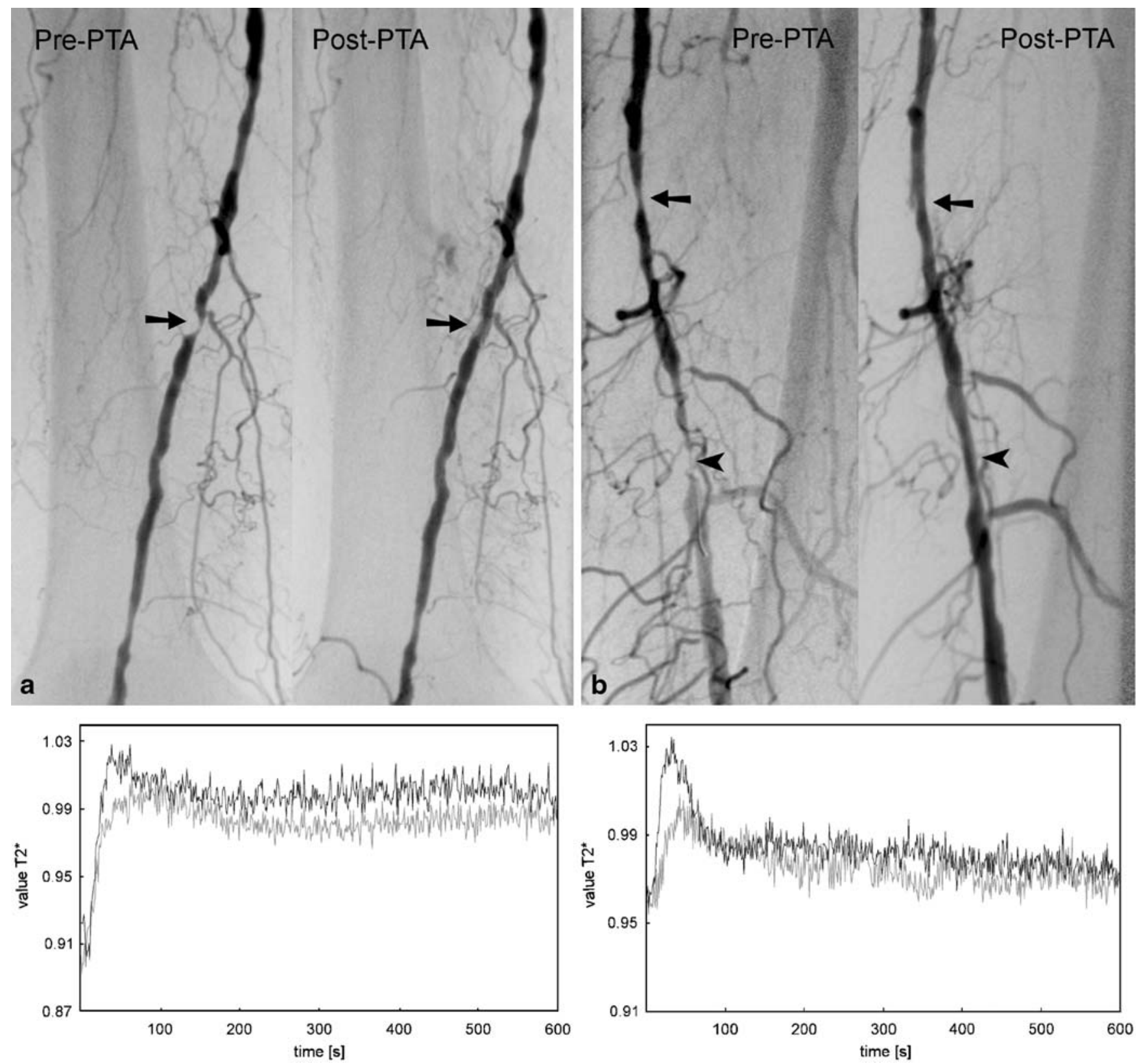

Fig. 2 a Top: An 80-year-old patient suffering from claudication intermittens. Diagnostic DSA pre- and post-PTA. A high-grade stenosis in the distal part of the SFA was successfully treated by antegrade PTA using a 5/40 balloon catheter. Bottom: Muscle BOLD response pre- (gray) and post-PTA (black). During reactive hyperemia, an accelerated $\mathrm{T} 2 *$ increase with earlier peaking and an enlarged maximum is observed for the post-interventional $\mathrm{T} 2 *$ timecourse. b Top: A 76-year-old patient suffering from claudication.

tion was observed between $\mathrm{BOLD}$ response and $\mathrm{TcPo}_{2}$ measurements [19].

Preexisting vasomotor relaxation is observed in PAOD patients, lowering the flow resistance along the peripheral arterial tree. BOLD signal changes are also dependent from blood volume [23]. Therefore, a regain of vasodilatation

Diagnostic DSA pre- and post-PTA. A high-grade tandem stenosis in the mid and distal part of the left SFA was successfully treated by antegrade PTA using a 5/40 balloon catheter. The post-interventional angiogram displays a non-significant residual stenosis of $<30 \%$. A clear reduction of the collateral pathway was observed. Bottom: Muscle BOLD response pre- (gray) and post-PTA (black). An accelerated TTP and an increase of T2* was observed for the postinterventional $\mathrm{T} 2 *$ time-course

properties can also contribute to an increase of BOLD amplitude $\left(\Delta \mathrm{T} 2{ }^{*}{ }_{\max }\right)$ leading to a higher blood inflow during reactive hyperemia after intervention. In the light of these physiological changes, a change of $11.1 \% \pm 3.6 \%$ to $12.3 \% \pm 3.8 \%$ is rather low and non-significant but detectable. 
The optimization of blood inflow also leads to an accelerated washout of the oxygenated blood from the calf muscle. Consequently, an acceleration of BOLD signal recovery to near baseline values can also be expected and is principally reflected by the lower EV levels in the PTAtreated group.

Compared with ABI values, changes of the BOLD key parameters are statistically not significant. This finding might be explained by the multifactorial influence of the BOLD response that is not only influenced by the macrovasculature but also from the microvascular network architecture, muscle fiber content and vessel angulation in the static field. We assume that these histological alterations do not change within 6 weeks after PTA treatment and might cover the perfusion-dependent BOLD response $[4,5,10,15,24,25]$.

The BOLD measurements are based on a post-occlusive reactive hyperemia paradigm using cuff-compression at midthigh level. The cuff-compression interrupts arterial inflow and venous outflow completely. Due to the fast cuff inflation within $5 \mathrm{~s}$, the contribution of venous filling to the BOLD effect was, therefore, kept as low as possible [26]. The application of the cuff-compression technique might be superior to treadmill paradigms, since it can be standardized and is independent on the patient's compliance [27].

There are some limitations of our study. First, the number of patients included is rather small. However, as a pilot study our major intent was to demonstrate an effect of PTA on muscle BOLD response by successful treatment. However, a trend was observed that underlines an optimization of blood inflow. A refinement of the measurement might be mandatory to increase the sensitivity of the method.
Secondarily, the patient collective is rather heterogeneous. Our inclusion criteria were based on claudication symptoms with high-grade stenosis of the SFA. However, we did not further differentiate between factors like number of non-significant stenoses along the arterial axis, number of crural arteries, blood pressure, venous and heart insufficiency. Furthermore, some patient were under antihypertensive drug treatment where a pharmacological influence on the arterial vessel wall and therefore on the BOLD signal change can be expected. Patients with critical limb ischemia (Fontaine Stage III) or demarcation of necroses (Fontaine Stage IV) were also excluded in this study because of the risk of progression of tissue loss due to the absolute ischemia caused by suprasystolic cuff compression.

It is presently unclear if optimization of MR parameters or pulse sequence might increase the sensitivity of this BOLD technique. A change from $1.5 \mathrm{~T}$ to higher static magnetic field strength is presumably another technical option to increase the sensitivity of muscle BOLD changes. This technical approach is presently under investigation $[28,29]$.

Our results are of interest because this is the first study demonstrating a change of BOLD response after successful PTA. Its impact on clinical management in patient treatment has to be investigated. However, further investigations are warranted, including other techniques like near infrared spectroscopy or nuclear magnetic resonance spectroscopy to elucidate the underlying multifactorial mechanism in more detail.

In conclusion, BOLD-MRI reveals changes of the key parameters T2* ${ }_{\text {max }}$, TTP, and EV after successful PTA of the calf muscles during reactive hyperemia.

\section{References}

1. Diehm C, Schuster A, Allenberg JR, Darius H, Haberl R, Lange S, Pittrow D, von Stritzky B, Tepohl G, Trampisch HJ (2004) High prevalence of peripheral arterial disease and comorbidity in 6880 primary care patients: cross-sectional study. Atherosclerosis 172:95-105

2. Lange S, Diehm C, Darius H, Haberl R, Allenberg JR, Pittrow D, Schuster A, von Stritzky B, Tepohl G, Trampisch HJ (2003) High prevalence of peripheral arterial disease but low antiplatelet treatment rates in elderly primary care patients with diabetes. Diabetes Care 26:3357-3358

3. Clyne CA, Mears H, Weller RO, O, Donnell TF (1985) Calf muscle adaptation to peripheral vascular disease. Cardiovasc Res 19:507-512
4. Henriksson J, Nygaard E, Andersson J, Eklof B (1980) Enzyme activities, fibre types and capillarization in calf muscles of patients with intermittent claudication. Scand J Clin Lab Invest 40:361369

5. Regensteiner JG, Wolfel EE, Brass EP, Carry MR, Ringel SP, Hargarten ME, Stamm ER, Hiatt WR (1993) Chronic changes in skeletal muscle histology and function in peripheral arterial disease. Circulation 87:413-421

6. Dormandy JA, Rutherford RB (2000) Management of peripheral arterial disease (PAD). TASC Working Group. TransAtlantic Inter-Society Consensus (TASC). J Vasc Surg 31:S1-S296

7. Stein R, Hriljac I, Halperin JL, Gustavson SM, Teodorescu V, Olin JW (2006) Limitation of the resting anklebrachial index in symptomatic patients with peripheral arterial disease. Vasc Med 11:29-33
8. Lebon V, Brillault-Salvat C, Bloch G, Leroy-Willig A, Carlier PG (1998) Evidence of muscle BOLD effect revealed by simultaneous interleaved gradient-echo NMRI and myoglobin NMRS during leg ischemia. Magn Reson Med 40:551-558

9. Lebon V, Carlier PG, Brillault-Salvat C, Leroy-Willig A (1998) Simultaneous measurement of perfusion and oxygenation changes using a multiple gradient-echo sequence: application to human muscle study. Magn Reson Imaging 16:721-729

10. Noseworthy MD, Bulte DP, Alfonsi J (2003) BOLD magnetic resonance imaging of skeletal muscle. Semin Musculoskelet Radiol 7:307-315 
11. Noseworthy MD, Kim JK, Stainsby JA, Stanisz GJ, Wright GA (1999) Tracking oxygen effects on MR signal in blood and skeletal muscle during hyperoxia exposure. J Magn Reson Imaging 9:814-820

12. Ogawa S, Lee TM, Kay AR, Tank DW (1990) Brain magnetic resonance imaging with contrast dependent on blood oxygenation. Proc Natl Acad Sci USA 87:9868-9872

13. Kwong KK, Belliveau JW, Chesler DA, Goldberg IE, Weisskoff RM, Poncelet BP, Kennedy DN, Hoppel BE, Cohen MS, Turner R, Cheng HM, Brady TJ, Rosen BR (1992) Dynamic magnetic resonance imaging of human brain activity during primary sensory stimulation. Proc Natl Acad Sci USA 89:5675-5679

14. Uludag K, Dubowitz DJ, Yoder EJ, Restom K, Liu TT, Buxton RB (2004) Coupling of cerebral blood flow and oxygen consumption during physiological activation and deactivation measured with fMRI. Neuroimage 23:148-155

15. Carlier PG, Bertoldi D, Baligand C, Wary C, Fromes Y (2006) Muscle blood flow and oxygenation measured by NMR imaging and spectroscopy. NMR Biomed 19:954-967

16. Donahue KM, Van Kylen J, Guven S, El-Bershawi A, Luh WM, Bandettini PA, Cox RW, Hyde JS, Kissebah AH (1998) Simultaneous gradient-echo/ spin-echo EPI of graded ischemia in human skeletal muscle. J Magn Reson Imaging 8:1106-1113
17. Toussaint JF, Kwong KK, M'kparu FO, Weisskoff RM, LaRaia PJ, Kantor HL, M, Kparu F (1996) Perfusion changes in human skeletal muscle during reactive hyperemia measured by echoplanar imaging. Magn Reson Med 35:62-69

18. Ledermann HP, Schulte AC, Heidecker HG, Aschwanden M, Jager KA, Scheffler K, Steinbrich W, Bilecen D (2006) Blood oxygenation leveldependent magnetic resonance imaging of the skeletal muscle in patients with peripheral arterial occlusive disease. Circulation 113:2929-2935

19. Ledermann HP, Heidecker HG, Schulte AC, Thalhammer C, Aschwanden M, Jaeger KA, Scheffler K, Bilecen D (2006) Calf muscles imaged at BOLD MR: correlation with $\mathrm{TcPO} 2$ and flowmetry measurements during ischemia and reactive hyperemiainitial experience. Radiology 241:477-484

20. Speck O, Hennig J (1998) Functional imaging by $\mathrm{I} 0$ - and $\mathrm{T} 2 *$-parameter mapping using multi-image EPI. Magn Reson Med 40:243-248

21. Hanna GP, Fujise K, Kjellgren O, Feld S, Fife C, Schroth G, Clanton T, Anderson V, Smalling RW (1997) Infrapopliteal transcatheter interventions for limb salvage in diabetic patients: importance of aggressive interventional approach and role of transcutaneous oximetry. J Am Coll Cardiol 30:664-669

22. Wagner HJ, Schmitz R, Alfke H, Klose KJ (2003) Influence of percutaneous transluminal angioplasty on transcutaneous oxygen pressure in patients with peripheral arterial occlusive disease. Radiology 226:791-797
23. Mandeville JB, Jenkins BG, Kosofsky BE, Moskowitz MA, Rosen BR, Marota JJ (2001) Regional sensitivity and coupling of BOLD and CBV changes during stimulation of rat brain. Magn Reson Med 45:443-447

24. Boxerman JL, Hamberg LM, Rosen BR, Weisskoff RM (1995) MR contrast due to intravascular magnetic susceptibility perturbations. Magn Reson Med 34:555-566

25. Damon BM, Gore JC (2005) Physiological basis of muscle functional MRI: predictions using a computer model. J Appl Physiol 98:264-273

26. Duteil S, Wary C, Raynaud JS, Lebon V, Lesage D, Leroy-Willig A, Carlier PG (2006) Influence of vascular filling and perfusion on BOLD contrast during reactive hyperemia in human skeletal muscle. Magn Reson Med 55:450-454

27. Berglund B, Eklund B (1981) Reproducibility of treadmill exercise in patients with intermittent claudication. Clin Physiol 1:253-256

28. Duong TQ, Yacoub E, Adriany G, Hu X, Ugurbil K, Kim SG (2003) Microvascular BOLD contribution at 4 and 7 $\mathrm{T}$ in the human brain: gradient-echo and spin-echo fMRI with suppression of blood effects. Magn Reson Med 49:1019-1027

29. Duong TQ, Yacoub E, Adriany G, Hu X, Ugurbil K, Vaughan JT, Merkle H, Kim SG (2002) High-resolution, spinecho BOLD, and CBF fMRI at 4 and 7 T. Magn Reson Med 48:589-593 